\title{
Healthcare Critical Infrastructure Stochastic Interdependencies Simulation Model for Smart Cities: Flood Disaster Scenario
}

\author{
Nivedita Nukavarapu $^{1, *}$, Surya Durbha ${ }^{1}$ \\ Centre of Studies In Resources Engineering, Indian Institute of Technology Bombay, Mumbai, India \\ nnivedita@iitb.ac.in, sdurbha@iitb.ac.in
}

Commission VI, WG III/IVe

KEYWORDS: Critical Infrastructure, Flood, Interdependencies, Stochastic Petri Net, IoT Flood Sensor, Spatio-Temporal, Disaster Preparedness

\begin{abstract}
:
Healthcare Critical Infrastructure (HCI) is not an independent network; the operation of a healthcare facility depends on many other Critical Infrastructure (CI) networks such as electric supply CI, water supply CI, etc., forming an interdependent CI network. During a flooding disaster event, as the flood levels rise, the interdependent HCI network becomes vulnerable. A failure in one of the CI results in failure of the dependent CI. During a disaster event such as flooding, the failures propagate and cause cascading failures like a domino effect. The paper proposes an IoT based flood sensor network integrated with a stochastic Petri net interdependent healthcare critical infrastructure network simulation model. An IoT (Internet of Things) based flood water level sensor network can deliver real-time information on the flood conditions at the various interdependent CI facilities in the interdependent network, using the Sensor Observation Services (SOS). The Stochastic Petri Net (SCPN) based interdependent Healthcare Critical Infrastructure (HCI) simulation model, is used to model and simulate the stochastic interdependencies between the interdependent HCI networks. The real-time flood sensor network is integrated with the SCPN based interdependent HCI simulation model. The end to end system is developed in a spatiotemporal environment. This kind of an integrated simulation model will help the end-user to understand system dynamics in real-time, visualize and predict the propagation of cascading failure scenarios in an Interdependent HCI network in a spatiotemporal environment, during a flooding scenario. Real-time information simulation would help disaster response personnel to respond to the question, 'what if something else happens?
\end{abstract}

\section{INTRODUCTION}

Critical Infrastructure (CI) refers to essential services such as water supply, power, transportation (road, rail, air, and water), and telecommunications systems, energy systems (electric power, oil, and gas), hospitals (Healthcare), finance, and government services. Sometimes also referred to as lifeline systems without which, emergency response systems and other infrastructure cannot operate as intended (Rinaldi, Peerenboom and Kelly, 2001) (Dudenhoeffer et al., 2006) (Ouyang, 2014). Urban Flooding can have severe implications on the Interdependent Critical Infrastructures at the onset of a flood.

Critical infrastructure networks are dependent on each other for their functioning. For example, a healthcare facility (hospital) is dependent on the power grid (electric substation) for electric supply and the water distribution network (water pumping station) for water supply. A failure in one of the CI results in failure of the dependent CI. During a disaster event, the failures propagate and cause cascading failures like a domino effect. During a flooding scenario, Healthcare Critical Infrastructure
(HCI) represents a critical and essential resource, and as the flood levels rise and the existing HCI facilities fiercely struggle to keep up with the pace, the underpreparedness of most cities becomes evident. As shown in Figure 1, healthcare critical infrastructure is affected in many ways.

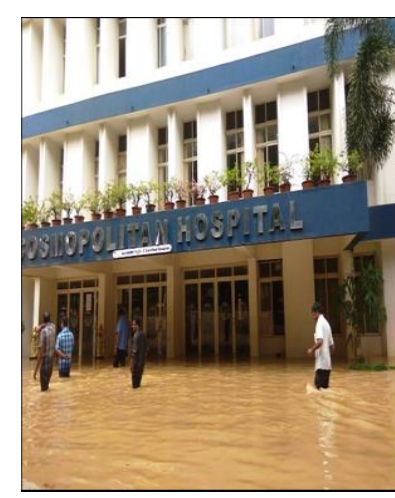

a) Inundated Hospital $\mathrm{CI}^{\mathrm{i}}$

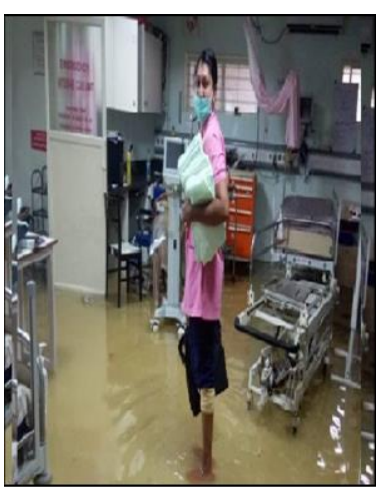

b) Inundated ICU in a Hospital $\mathrm{CI}^{\mathrm{ii}}$
Figure 1. Inundated hospital facility and inundated ICU in an HCI facility during a flood

\footnotetext{
* Corresponding author
} 
Due to the inundation of the HCI facility and disruption in the services from other CI such as electricity supply and water supply, the HCI facility results in a disastrous situation (Nukavarapu, Durbha,2016) (Nukavarapu, Durbha, 2017). Instead of getting to a state of emergency, one in which interdependent HCI facilities network becomes vulnerable. There is an urgent need for smarter cities where we could prevent and prepare our critical infrastructure for the challenges ahead (Loosemore, Chow, McGeorge, 2014) (Nukavarapu, Durbha, 2016).

\subsection{Motivation}

Flood warning systems are already in place and are widely used for monitoring and forecasting flood disasters. However, with the concept of smart city utilizing smart internet of things technologies (IoT), the flood warning system can be made even more effective.

Real-time flood warning based applications will help predict the increase in the flood water level at a CI facility location (such as a hospital, water pumping station, electrical substation, roads, etc.). An IoT based flood water level sensor network integrated with the interdependent HCI network can deliver the real-time information on the flood conditions at the various critical infrastructure $(\mathrm{CI})$ facilities.

The main objective of this work is to develop a real-time situational awareness simulation model for an interdependent HCI network, integrated with an IoT based flood sensor network in a spatiotemporal environment. The flood level at the various critical infrastructure (CI) facilities is collected by an IoT based flood sensor network. The flood sensor network consists of different IoT based flood level sensors placed at the various CI facilities.

The real-time information is sent to a simulation model using the sensor observation services (SOS). The sensor information consisting of the location id, timestamp, flood level, etc., with other geographical attributes is sent to a Stochastic Coloured Petri Net (SCPN) based interdependent HCI simulation model, which is set in a spatiotemporal environment. The end to end system helps the decision-maker in visualizing the inundation of various CI facilities and propagation of cascading failures in real-time.

\section{2 "What if" Disaster Scenario}

CI facilities can be represented as nodes in a network where they are connected through a set of links depicting the logical relationship among them; these nodes are interdependent and interact with each at other at various levels, such that the state of each infrastructure influences or is correlated to the state of another. In a scenario such as the disruption in the service of one infrastructure nodes (e.g. transportation) of the network during a disaster would lead to cascading and escalating disruptions across other infrastructures nodes (schools, hospitals, power grid, etc.) in the network. Given below is a What-if scenario depicting the kind of dependencies that are encountered by an HCI facility in a flood situation;

"The IoT based flood level sensor at an electric supply CI facility (electric substation 'A') can send alerts about the failure of the substation operation due to high floodwater level. Hospital ' $A$ ' is dependent on the electrical substation A, for electric supply. The IoT flood sensor at the water pumping station sends alerts about its inundated state. On receiving these alerts hospital ' $A$ ' can predict using the simulation model, an estimate of the time of disruption of electrical supply and water supply, and check for an alternative back up. Also, the hospital checks for the time estimate of its inundated state, due to increased flood levels and makes arrangements for evacuation of patients".

The end-user can query from the end to end system about the various states of the flooding event such as ;

- What areas around the Healthcare facility have submerged?

- What is the existing Flood Water Level at various Critical infrastructure facilities?

- What is the shortest and safest route for emergency evacuation of patients from the Hospital?

- If a failure occurs in one of the critical infrastructures such as a failure at an electric substation in an electric supply CI network, what would be the cascading effect on the healthcare $\mathrm{CI}$ ?

The ability to have a real-time simulation model, considering the above scenario, can have an impact on the decision making heuristics during a flood event. The simulation model will be able to visualize, predict, and analyze the system dynamics in real-time, and predict the propagation of cascading failure scenarios in an interdependent HCI network during a flooding event (Nukavarapu, Durbha, 2016) (Nukavarapu, Durbha, 2017).

\section{BACKGROUND}

Petri net is based on graph theory and is one of the best tools to model discrete events dynamic systems. The stochastic interdependencies between the various critical infrastructure facilities can be modeled using Generalized Stochastic Petri Net (GSPN) (Ouyang, 2014) (Street, Desrochers, 2003) (Krings, Oman, 2003) (Gursesli, Desrochers, 2004) (Sultana, Chen, 2009) (Bobbio, 2011). 
The main limitation of these models is that they cannot be used to model, simulate, and analyze the dynamics of the uncertainty of a large complex system. This limitation is addressed by developing a simulation model using Stochastic Coloured Petri Net (SCPN), which increases the strength of the Petri Net and makes it possible to simulate a large real-time complex process.

Coloured Petri Net (CPN) (Jensen, 1987) is a graphical modeling language that is used to model complex systems and then analyze the states of the system and the events that change the system from one state to another. CPN is a combination of Petri net and programming languages; hence, it is also sometimes referred to as a high-level Petri net. Petri net provides the primitives for process interaction; while the programming language provides the primitives for the definition of data types and the manipulation of data values (Jensen, Kristensen, 2009) (Jensen, 1996) (Jensen, 2005) associated with arcs. The real-time data from the flood sensors are sent to the Stochastic Coloured Petri net (CPN) model using sensor observations services (explained in detail in section 3 ).

Zhou et al. (2018) proposed an intelligent token PN for the modeling and control of the systems (Zhou, Reniers, 2018). Through their model, tokens that denoted job instances can carry real-time information about system status and move just like the real-life objects. Zhang et al. (2015) used the hierarchical-timed coloured Petri net (HTCPN) model to analyze the real-time production events, where the events were extracted according to a multi-level event model (Zhang et al., 2016). Kuo et al. (2009) proposed a decentralized PN based wireless sensor node architecture (PN-WSNA), which can be used to model and implement a reconfigurable sensor network (Kuo and Siao, 2009). Boubeta-Puig et al. (2019) proposed a complex event process (CEP) and Petri Nets to monitor air pollutants (Boubeta-Puig et al., 2019).

A Coloured Petri Net is defined as a 6-tuple $C P N=(\mathrm{P}$, T, I, O, $\mathrm{M}_{0}$ ), where $P=\{P 1, P 2, P 3 \ldots P n\}$ is finite and non-empty places (conditions or states of the network); $T$ $=\{T 1, T 2, T 3 \ldots T n\}$ is finite and non-empty transitions (events in the network). $P \cup T \neq \varnothing, P \cap T \neq \emptyset, I: P \times T \rightarrow N$ denotes the inputs, which defines all the directional arcs from place to transition, where $N=\{0,1,2 \ldots\}$; input and output functions associated with arcs. $M: P \rightarrow N$ denotes the marking of the Petri net, which defines the number of tokens present in the places, $M 0$ is the initial Marking. $C$ is a color set function that assigns a color set to each place.

The transition fires by removing tokens from its input places and creates tokens that are distributed to its output places. Firing can continue as long as there exists at least one enabled transition. Otherwise, the execution will be stopped (Jensen, 1987) (Jensen, Kristensen, 2009) (Jensen, 1996).
Coloured Stochastic Petri Net (CSPN) extends the Coloured Petri net where the transitions fire after a probabilistic delay determined by a random variable, as shown in Figure 2. Tokens can move from one place to another by crossing enabled transitions, after a random sojourn time (defined by the probability distributions of transitions and by input functions) in the input place (Jensen, Kristensen, 2009).

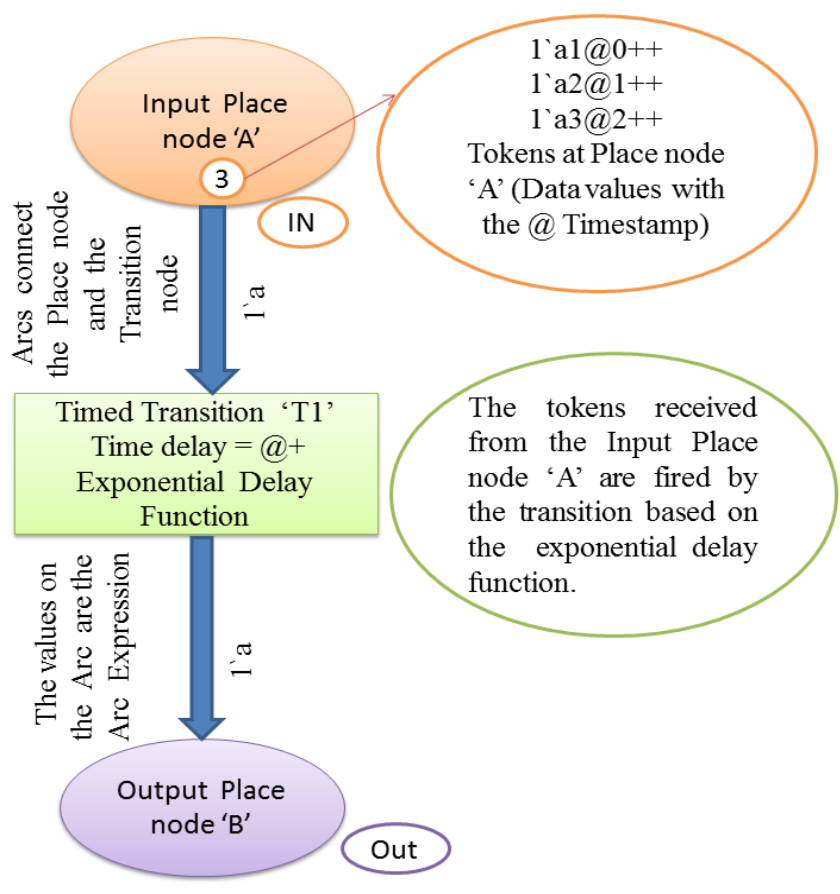

Figure 2. Example of a Stochastic Coloured Petri Net

\section{ARCHITECTURAL FRAMEWORK}

An IoT based flood sensor network integrated with Interdependent Healthcare Critical Infrastructure network Stochastic Petri net simulation model (HCISCPN) is described by the concept of different states of the simulation model based on the real-time IoT data tokens form the various IoT sensors at the CI facilities. The HCISCPN simulation model changes to a new state based on the IoT flood sensor data. The Architectural Framework for IoT based flood sensor network integrated with interdependent healthcare CI network stochastic Petri net simulation model is shown in Figure 3.

It constitutes two Coloured Petri Net (CPN) models, the IoT based flood water level sensor network coloured Petri net (CPN) model and the interdependent healthcare critical infrastructure network stochastic Petri net simulation model. The CPN models are explained in detail in the following sections. The entire end to end system is developed in a spatiotemporal environment, by integrating the entire system with a Geographic information system. 


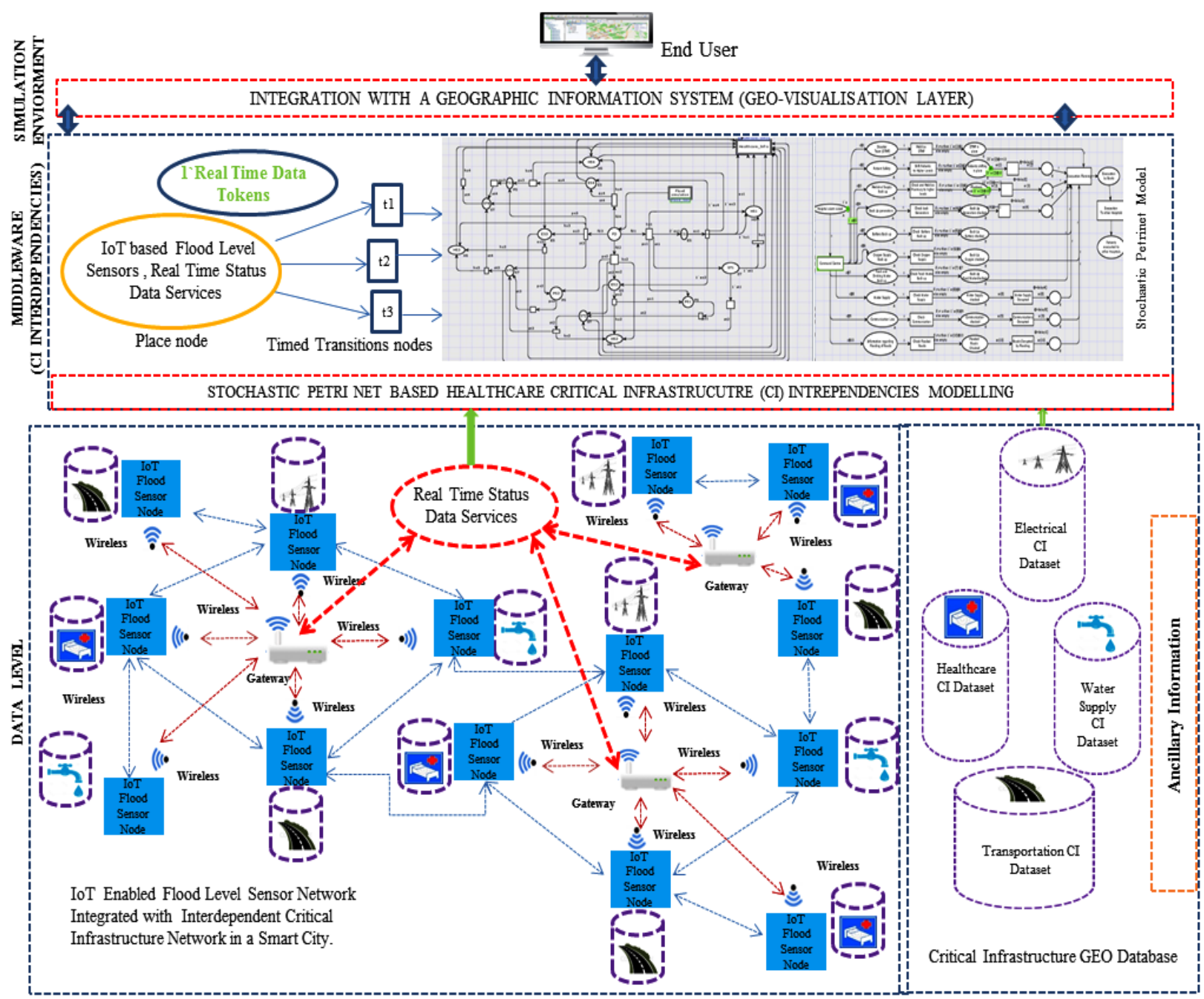

Figure 3. Architecture for IoT based flood sensor network integrated with stochastic Petrinet based interdependent healthcare CI network simulation model

\subsection{Development of IoT Based Flood Water Level sensor Network Coloured Petri net (CPN) model}

We have considered a scenario where a set of flood level sensor stations in an urban city are placed at different CI facilities. These sensors take flood water level reading each minute, and send the data to a central monitoring system. The interdependent critical infrastructure network will be warned when the following categories of four different flood levels are observed (These categories are used by NOAA ${ }^{\text {iii }}$ to differentiate different flood levels).

We have assigned a maximum flood level for each stage; as the flood level reaches its maximum level the category of the flood changes.

a) Action Stage: At this stage, the water level is slightly above the river or lake banks. We have considered this stage when the water level sensor detects the first reading greater than $1 \mathrm{ft}$. b) Minor flood Stage: The flooding has just started at this stage; roads, marshland, etc., will be inundated with water. The flood level sensor readings for floodwater higher than $2 \mathrm{ft}$.

c) Moderate flood Stage: Buildings and different CI facilities might get inundated at this stage. roads are likely to be closed, and some low-lying areas completely inundated is likely. Most of the CI facilities would be partially submerged. Large-scale evacuations may be necessary. To activate this stage, the flood level sensor readings during this stage are greater than $5 \mathrm{ft}$.

The flood level information from the Flood Level (FL) sensors web is integrated in real-time with the interdependent HCI network using the Sensor Observation Service (SOS) ${ }^{\text {iv }}$, SOS is an interoperable web service based on the OGC (Open Geospatial Consortium) $)^{\mathrm{v}}$ standards. The standardized web service would help in querying real-time flood level sensor data and FL sensor data time series. 
In an IoT based flood water level sensor network CPN as shown in Figure 4, flood level sensor place is used to represent the status of the sensor and collect real-time flood water level information. When a flooding event happens in the sensing area of a sensor, the related sensor place will obtain a token that carries the real-time status of the activity. The FL sensor data comprises descriptions of FL sensors themselves, which are encoded in the Sensor Model Language (SML) ${ }^{\mathrm{vi}}$, and the flood level measured values are in the observations and measurements encoding format reading, Sensor station $I D$, Location ID, and its attribute. Attributes denote the sensing information (e.g., real-time flood water level captured by the IoT based flood water level sensor). The CPN model checks the sensor's status: if the sensor cannot read flood water level data, the 'No Sensor Reading' place will obtain a token.

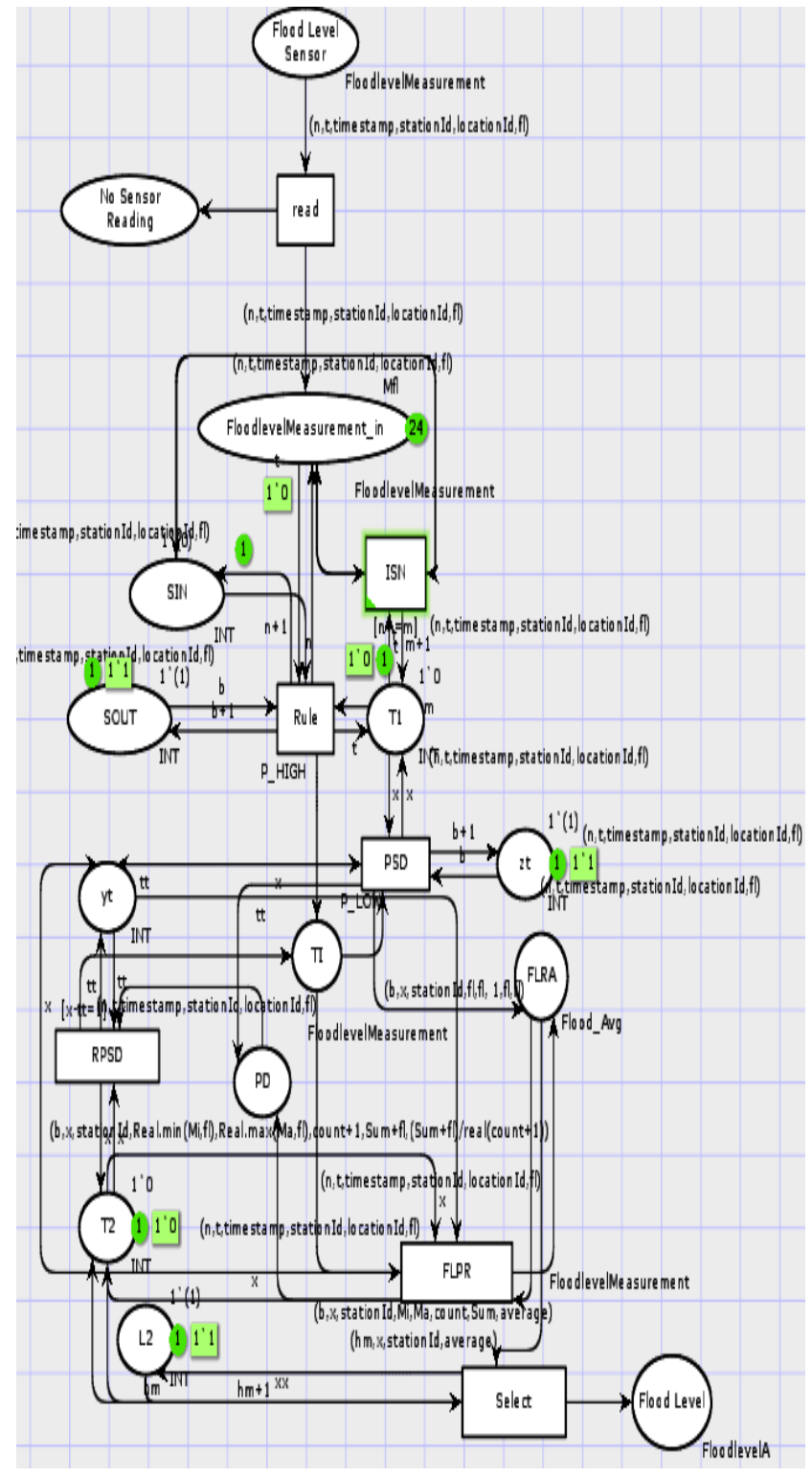

If the sensor reads the real-time event data 'Flood Level Measurement' place node in the Petri net simulation model will receive the data token. The flood level sensor with Station ID takes continues reading of the water level, for every 10 minutes. The system checks if the flood level is higher than $1 \mathrm{ft}$ in the sensor readings and if it exists the CPN will compute the count, maximum, minimum, sum, and the average of all the readings values taken in the last 10 minutes and will group the information by each station. Depending on the average of the floodwater level sensor reading values, the $\mathrm{CI}$ facilities in the interdependent CI network are notified about the flood stage. For example, if the average flood water level is around $3 \mathrm{ft}$, the flood category is decided as the Major Flood Stage. And the patients from a healthcare CI facility have to be evacuated to a safer healthcare facility.

\subsection{Development of Interdependent Healthcare Critical Infrastructure Network CPN simulation model}

The output from the IoT based flood water level sensor network CPN is used to trigger the events in the interdependent HCI network CPN model, as shown in Figure 5. The affected nodes (i.e. hospitals, electric substations, water storage tanks, and pumping stations) of the healthcare CI network; electric supply CI and water supply CI respectively can be identified. Following is an example in the scenario which has been considered while constructing the cascading effect of a failure of one CI on the other CI in the simulation model.

Disruption of electric substation node (es1) would affect the electric substation place node (es2) which would later affect the working of pumping station node (ps1), disruption of which affects the water storage tank place node (st2), which in return affects the water supply for hospital place node (hs1). When the category of the flood is being declared and then there is an alarm notification indicating the flooding of the hospital place node (hs1), failure of electric supply from electric substation place node (es1), and also the failure of water supply from the water pumping station place node (ps1) to the hospital place node (hs1).

The command center designated activities would be performed priority wise which includes; Alert and mobilizes the hospital staff, organizes the medical equipment for evacuation, alert the ambulance services, alert the patient wards which need to be evacuated respectively, and later evacuating the patients to a nearby unaffected hospital. For example, if the hospital (hs1) is the affected hospital then the patients can be evacuated to the hospital (hs2) (Nivedita and Durbha, 2014).

Figure 4. IoT based flood water level sensor network CPN model 


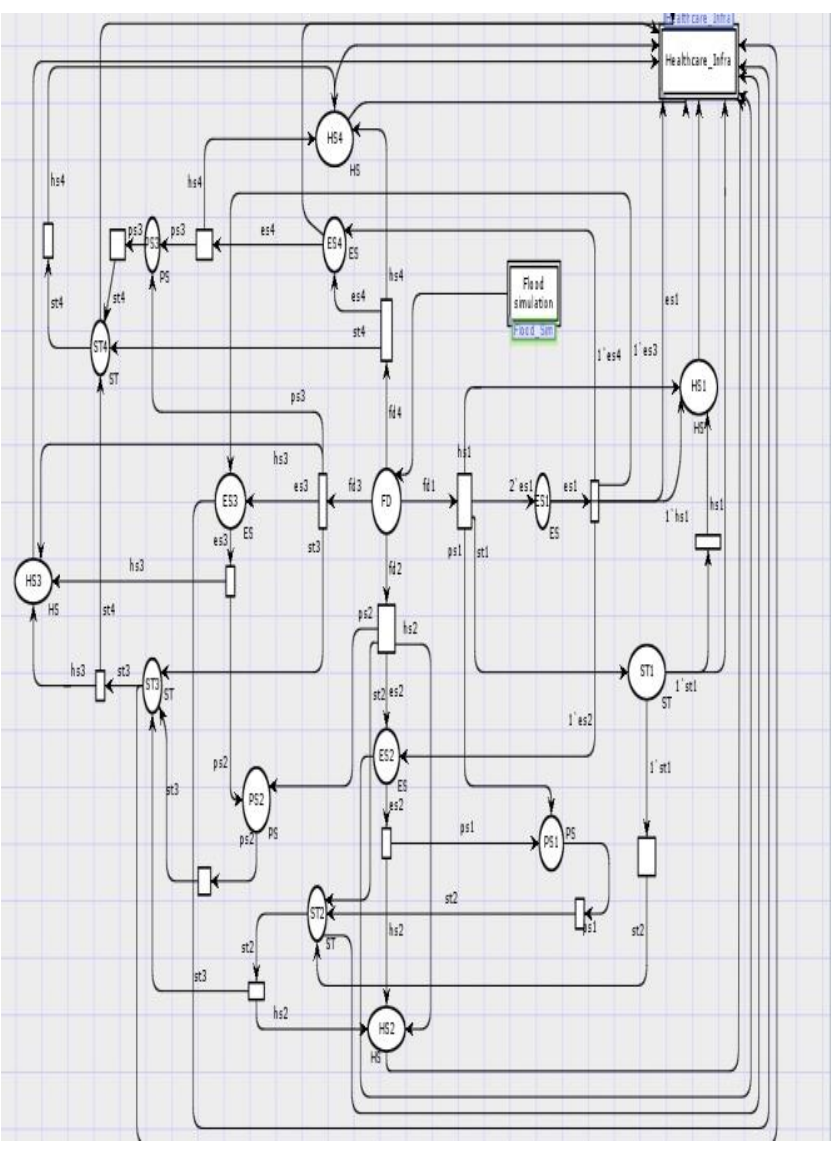

Figure 5. Interdependent Healthcare Critical Infrastructure Network CPN (Nivedita and Durbha, 2014)

\subsection{Spatio-Temporal Environment}

The end to end system IoT flood sensor network integrated with the HCISCPN simulation model is integrated with a geographic information system which provides visualization on a spatiotemporal scale and helps the user in decision making. The various place nodes (locations) in the HCISCPN simulation model are registered with critical infrastructure geodatabases having a set of properties according to its categories, such as healthcare infrastructure $\mathrm{CI}$, transportation $\mathrm{CI}$, water supply CI, etc., and the IoT based flood water levels sensor network location information such as sensor stations ID and location ID. Thus providing the simulation model with GIS related information.

The directed arc from the place nodes and transition nodes of the HCISCPN simulation model depicts the interdependencies among the CIs. In this simulation model, tokens representing the flood level instances carry real-time knowledge about the system. The real-time information can be gathered from the various sensors that are installed at the Critical Infrastructure Facilities at different instances of time. So for every real-time data instance, the performance of the HCISCPN simulation model would behave differently (Nukavarapu and Durbha, 2017).
A JAVA ${ }^{\text {vii }}$ code is developed by using CPN tools ${ }^{\text {viii; }}$ a java extension is known as Comms/CPN and Geo-Tools ${ }^{\mathrm{ix}}$, which is an open-source Java package for the integration of the coloured Petri net simulation model and a GIS system. Comms/CPN makes it possible for CPN Tools to communicate based on TCP/IP with external applications and processes. Geo-Tools provide standards compliant methods for the manipulation of geospatial data, for example, to implement Geographic Information Systems (GIS).

Spatial data layers such as road network (transportation CI), healthcare facility network (location of healthcare facilities), water supply network (Water bodies, geographic area, and water pumping station nodes), electric supply network (location of electric substation), and also flood level sensor network (location of flood level sensors) are visually combined resulting in a complex spatial data set (Nukavarapu, Durbha, 2017).

The Spatio-temporal visual analytics in the dynamic simulation model based on real-time information enables the users to investigate data features over time using a visual display based on geographic maps as shown in Figure 6. And also visualize and analyze the impact of the disaster depending on its severity stage.

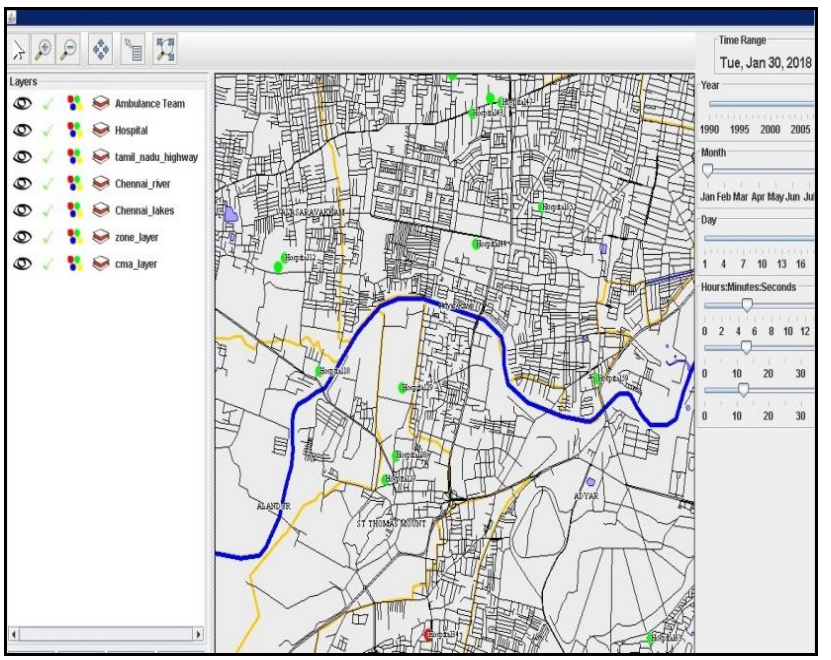

Figure 6. Geo-visualization for IoT flood sensor network integrated with Interdependent HCI SCPN Simulation Model

\section{Conclusion}

This paper presents a novel approach of IoT based flood sensor network integrated with interdependent healthcare CI network stochastic Petri net simulation model. Using the flood-related information across the Internet of Things (IoT) platforms is a way of making cities better equipped to face a flooding situation. The ability to have real-time flood level data information integrated with an interdependent ci network simulation 
model can simulate the cascading failure that can unfold during a flood disaster.

Our key contribution is the framework of the approach and the design of the IoT Based Flood sensor Network Integrated with Interdependent Healthcare CI Network Stochastic Petri net Simulation Model during a flood event. The state-space analysis of the simulation model helps in answering the queries in real-time as shown in the example. For example; what is the optimum time taken by a CI facility to be incomplete failure (nonoperational) state due to flooding?

This will help the is the disaster response personnel or organizations managing crisis activities (e.g. Health care managers, Emergency management personnel, etc.) to manage disaster challenges and develop response activities accordingly and be able to prepare contingency plans based on it. Real-Time information simulation would help them to respond to the question, 'what if something else happens?'

\section{REFERENCES}

Bobbio, A. (2011) 'System Modelling with Petri Nets', in. doi: 10.1007/978-94-009-0649-5_6.

Boubeta-Puig, J. et al. (2019) 'MEdit4CEP-CPN: An approach for complex event processing modeling by prioritized colored petri nets', Information Systems. doi: 10.1016/j.is.2017.11.005.

Dudenhoeffer, D. et al. (2006) 'Critical Infrastructure Interdependency Modeling: A Survey of Critical Infrastructure Interdependency Modeling: A', Contract. doi: $10.2172 / 911792$.

Gursesli, O. and Desrochers, A. A. (2004) 'Modeling infrastructure interdependencies using Petri nets', in. doi: 10.1109/icsmc.2003.1244625.

Jensen, K. (1987) 'Coloured petri nets', in Lecture Notes in Computer Science (including subseries Lecture Notes in Artificial Intelligence and Lecture Notes in Bioinformatics). doi: 10.1007/978-3-540-47919-2_10.

Jensen, K. (1996) Coloured Petri Nets: Basic Concepts, Analysis Methods and Practical Use, Media. doi: 10.1109/ICEICE.2011.5777259.

Jensen, K. (2005) 'Sixth Workshop and Tutorial on Practical Use of Coloured Petri Nets and the CPN Tools', (October), p. 300 .

Jensen, K. and Kristensen, L. M. (2009) Coloured Petri Nets: Modelling and validation of concurrent systems, Coloured Petri Nets: Modelling and Validation of Concurrent Systems. doi: 10.1007/b95112.

Krings, A. and Oman, P. (2003) 'A simple GSPN for modelling common mode failures in critical infrastructures', in Proceedings of the 36th Annual Hawaii International Conference on System Sciences, HICSS 2003. doi:

\subsection{9/HICSS.2003.1174908}

Kuo, C. H. and Siao, J. W. (2009) 'Petri net based reconfigurable wireless sensor networks for intelligent monitoring systems', in Proceedings - 12th IEEE International Conference on Computational Science and Engineering, CSE 2009. doi: 10.1109/CSE.2009.252.

Loosemore, M., Chow, V. and McGeorge, D. (2014) 'Managing the health risks of extreme weather events by managing hospital infrastructure', Engineering, Construction and Architectural Management, 21(1), pp. 4-32. doi: 10.1108/ECAM-10-20120060 .

Nukavarapu, N. and Durbha, S. (2016) 'Stochastic coloured petrinet based healthcare infrastructure interdependency model', in International Archives of the Photogrammetry, Remote Sensing and Spatial Information Sciences - ISPRS Archives. doi: 10.5194/isprsarchives-XLI-B8-223-2016.

Nukavarapu, N. and Durbha, S. (2017) 'GEO-Visual analytics for healthcare critical infrastructure simulation model', in International Geoscience and Remote Sensing Symposium (IGARSS). doi: 10.1109/IGARSS.2017.8128402.

Ouyang, M. (2014) 'Review on modeling and simulation of interdependent critical infrastructure systems', Reliability Engineering \& System Safety. doi: 10.1016/j.ress.2013.06.040.

Rinaldi, S. M., Peerenboom, J. P. and Kelly, T. K. (2001) 'Identifying, understanding, and analyzing critical infrastructure interdependencies', IEEE Control Systems Magazine. doi: 10.1109/37.969131.

Street, I. O. and Desrochers, A. A. (2003) 'Using Petri', Systems Engineering.

Sultana, S. and Chen, Z. (2009) 'Modeling flood induced interdependencies among hydroelectricity generating infrastructures', Journal of Environmental Management. doi: 10.1016/j.jenvman.2009.05.019.

Zhang, Y. et al. (2016) 'IoT-Enabled Real-Time Production Performance Analysis and Exception Diagnosis Model', IEEE Transactions on Automation Science and Engineering. doi: 10.1109/TASE.2015.2497800.

Zhou, J. and Reniers, G. (2018) 'Petri-net based evaluation of emergency response actions for preventing domino effects triggered by fire', Journal of Loss Prevention in the Process Industries. Elsevier, 51(November 2017), pp. 94-101. doi: 10.1016/j.jlp.2017.12.001.

\footnotetext{
${ }^{\mathrm{i}}$ www.thenewsminute.com

ii www.thenewsminute.com

iii https://www.noaa.gov/

${ }^{\text {iv }}$ https://www.opengeospatial.org/standards/sos

${ }^{v}$ https://www.opengeospatial.org/

vi https://www.opengeospatial.org/standards/sensorml

vii www.java.com

viii www.cpntools.com

${ }^{\mathrm{ix}}$ www.geotools.org
} 